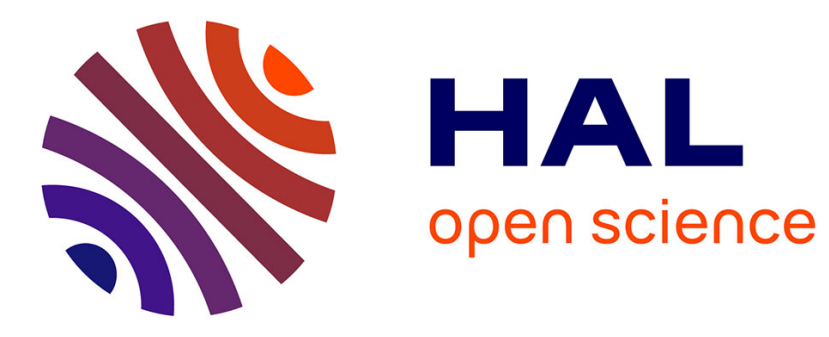

\title{
Transient signal characterization using multi-lag phase space analysis
}

Angela Digulescu, Cindy Bernard, Elena Lungu, Ion Candel, Cornel Ioana, Gabriel Vasile

\section{- To cite this version:}

Angela Digulescu, Cindy Bernard, Elena Lungu, Ion Candel, Cornel Ioana, et al.. Transient signal characterization using multi-lag phase space analysis. COMCAS 2015 - 2015 IEEE International Conference on Microwaves, Communications, Antennas and Electronic Systems, Nov 2015, Tel-Aviv, Israel. 10.1109/COMCAS.2015.7360411 . hal-01447978

\section{HAL Id: hal-01447978 https://hal.science/hal-01447978}

Submitted on 6 Mar 2017

HAL is a multi-disciplinary open access archive for the deposit and dissemination of scientific research documents, whether they are published or not. The documents may come from teaching and research institutions in France or abroad, or from public or private research centers.
L'archive ouverte pluridisciplinaire HAL, est destinée au dépôt et à la diffusion de documents scientifiques de niveau recherche, publiés ou non, émanant des établissements d'enseignement et de recherche français ou étrangers, des laboratoires publics ou privés. 


\title{
Transient signal characterization using multi-lag phase space analysis
}

\author{
Angela Digulescu ${ }^{1,2}$, Cindy Bernard ${ }^{1}$, Elena Lungu ${ }^{3}$, Ion Candel $^{1}{ }$, Cornel Ioana ${ }^{1}$, Gabriel Vasile $^{1}$ \\ ${ }^{1}$ Gipsa-lab, Grenoble Institute of Technology, 11 rue de Mathématiques, BP 46, \\ Saint Martin d'Hères, 38402, France \\ ${ }^{2}$ Military Technical Academy, 34-39 George Cosbuc Avenue, Bucharest, Romania \\ ${ }^{3}$ SIGINTEC, 334 rue Tour de l'Evêque, 30000, Nîmes, France \\ \{angela.digulescu, cindy.bernard, cornel.ioana\}@gipsa-lab.grenoble-inp.fr, elungu@ sigintec.fr
}

\begin{abstract}
Transient signals have proved to be difficult to characterize/classify because of their short duration and broad spectrum. Methods based on time-frequency analysis are used for transient detection, but their characterization is limited when it comes to similar signals that have close amplitudes and frequencies. This paper presents the multi-lag phase space analysis, an alternative to the classical approaches for the characterization of transient signals. New tools developed for this method are applied on multi-path acoustic signals which are acquired using an acoustic microphone placed in the center of a facility surrounded by walls. The results are compared with the ones obtained using time-frequency analysis.
\end{abstract}

Index Terms-Phase space, transient, recurrence, lag.

\section{INTRODUCTION}

Multi-path propagation implies that the received signal is the sum of the attenuated and delayed versions of the emission. Interference and phase shifting are a common occurrence in signal processing application, the effects of which can have a negative or positive effects on the results. When the source is represented by an electrical arc coming from a photovoltaic panel, it is important to classify the multi-path recorded signals in order to insure the safety an reliability of the system. While the detection of these faults was successfully achieved using the time-frequency analysis [1], their characterization arises a great interest for the classification of these signals in terms of system's monitoring [2].

The multi-lag phase space analysis is based on the phase space representation concept and it describes the evolution of a dynamic system in a multidimensional space, namely its phase space for multiple lags. Reference [3] presents the capability of discrimination between two similar signals using their evolution in the phase space.

Furthermore, new tools have been developed based on the multi-lag phase space analysis. The method is based on the evolution of a signal in a multi-dimensional phase space which creates a trajectory [4], [5]. This trajectory is described using several parameters (extreme points, area, position vector, angle, etc.) that change with the lag. In this paper, we present a new technique for the characterization of transient signals based on the information provided by the trajectory and its defining parameters. We will prove that it is possible to distinguish between multi-path acoustic signals using these tools .

The paper is organized as follows: section II describes the concept of phase space representation, section III presents the new tools introduced for the multi-lag analysis and section IV shows the results obtained using the proposed method. Section $\mathrm{V}$ gives further interpretation of the results and points out our future work.

\section{PHASE SPACE REPRESENTATION}

The concept of phase space representation comes from the dynamical systems theory [4] describing the evolution of a system in order to emphasize the states where the system returns. This evolution is highlighted by the trajectory obtained in the phase space. The trajectory is determined only by the measurement made on the system. For this reason, the multi-lag phase space analysis is a data-driven method which is a major advantage, because it does not need any prior information about the system.

A received signal is considered a measurement expressed as the time series[5] from eq. (1).

$$
\mathbf{s}=\{s[1], s[2], \ldots, s[N]\}
$$

The time series is, then, represented in a $m$ dimensional phase space. In this phase space, the values of the time series become the coordinates of the vectors represented:

$$
\overrightarrow{v_{[i]}}=\sum_{k=1}^{m} s[i+(k-1) \tau] \cdot \overrightarrow{e_{k}}, i=\overline{1, M}
$$

where $\overrightarrow{v_{[i]}}$ are the phase space vectors, $m$ is the embedding dimension of the phase space, $\tau$ is the delay (lag) between the samples, $M=N-(m-1) \tau$ and $\overrightarrow{e_{k}}$ are the axis unit vectors. Plotting these vectors in the phase space gives the trajectory of the system and each intersection of this trajectory represents a recurrence point.

The multi-lag phase space analysis can illustrate the evolution of the signal in any dimension, however we consider the representation of the signal in two dimensions, in order to simplify the interpretation of the results. Nevertheless, if the studied signals are more complex and a higher dimension 
would be more suitable, yet the $2 \mathrm{D}$ representation would be just the projection of those signals and its analysis would stand meaningful.

Hereby, considering the Cartesian coordinate system, the phase space points are given by:

$$
\begin{cases}x_{n} & =s[n] \\ y_{n} & =s[n+\tau]\end{cases}
$$

where $n$ is the $n$-th sample of the mesured time series and $\overrightarrow{v_{[n]}}=x_{n} \cdot \vec{i}+y_{n} \cdot \vec{j}$.

We recall several proprieties of this representation, as mentioned in [3]. Considerind the signals $s, s_{1}, s_{2}$ and $s_{3}$ with the following correspondence:

$$
\begin{aligned}
& s_{1}[n]=s[n+\delta] \\
& s_{2}[n]=s[\alpha n] \\
& s_{3}[n]=\beta \cdot s[n]
\end{aligned}
$$

where $\alpha, \beta$ and $\delta$ are constant that modifie the evolution of $s$ signal, the subsequent properties arise:

$$
\begin{aligned}
& \overrightarrow{v_{1[i]}}=\overrightarrow{v_{[i+\delta]}} \\
& \overrightarrow{v_{2[i]}}=\overrightarrow{v_{[\alpha i]}} \\
& \overrightarrow{v_{3[i]}}=\beta \cdot \overrightarrow{v_{[i]}}
\end{aligned}
$$

Based on these properties, the multi-lag phase space representation allows us to emphasize the similarities between signals as those from (4), their trajectory being invariant to translation and directly related to the lag and amplitude [3], [6], [7].

Thus, the unique representation of the trajectory depends on the lag and it is necessary to develop new tools in order to characterize the trajectory on the phase space. These tools are needed for a complete description and comparison between similar signals.

\section{NEW TOOLS FOR MULTI-LAG PHASE SPACE ANALYSIS}

In order to point out the properties for the newly developed tools, we consider the test signals similar to the ones used in [3].

Let the signal $x_{(\omega, \alpha, \varphi)}[n]$ be a generic signal given by eq. (6). Hereby, we consider two similar signals $s_{\left(\omega_{1}, \alpha_{1}, \varphi_{1}\right)}[n]$ and $s_{f\left(\omega_{2}, \alpha_{2}, \varphi_{2}\right)}[n]$, respectively where $\omega_{1} / \omega_{2}=1.0256, a_{1} / a_{2}=$ 0.667 and $\varphi_{1}-\varphi_{2}=-\pi / 3$. In addition, a third signal is considered, $s_{d}[n]$ which is the dilated version of $s[n]$ with $\alpha=2$ according to eq. (4).

$$
x_{(\omega, \alpha, \varphi)}[n]= \begin{cases}\cos [\omega n+\varphi] \cdot \exp [-a n], \text { for } & n=\overline{1, N}, \\ 0 & \text { otherwise }\end{cases}
$$

Figure 1 shows $s_{d}[n]$ is the dilated version of $s[n]$, having the same duration and amplitude, while $s_{f}[n]$ seems to be similar to $s[n]$. In [3], the wavelet approach has shown that these signals can not be set apart, but using the multi-lag phase

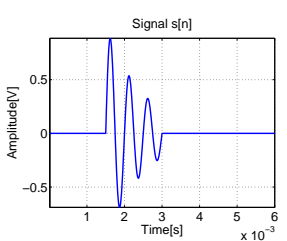

(a)

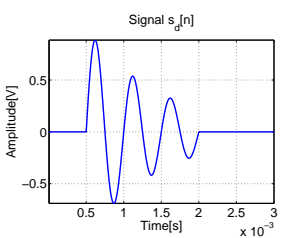

(b)

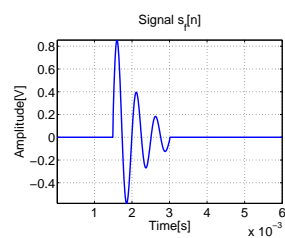

(c)
Figure 1: Signals used for highlighting the properties of multilag phase space analysis: (a) $s[n]$, (b) $s_{d}[n]$, (a) $s_{f}[n]$,

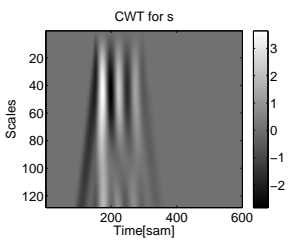

(a)

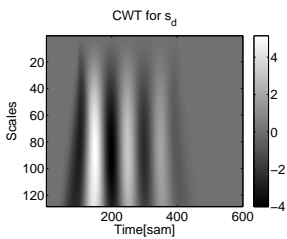

(b)

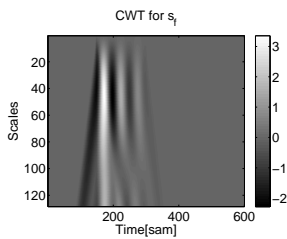

(c)
Figure 2: Continuous wavelet transform (using Haar mother wavelet function) the signals used to highlight the properties of multi-lag phase space analysis

space representation the distinction can be made. Figure 2 shows the lack of information that can be obtained using the wavelet approach for the signals previously considered.

The main concept of the multi-lag approach is presented in fig. 3 .

The first tool proposed represents the extreme points on the trajectory. We consider four possible extreme points on the trajectory:

$$
\begin{cases}A\left(x_{k A}, y_{k A}\right), & x_{k A}=\max _{n}\left(x_{n}\right) \\ B\left(x_{k B}, y_{k B}\right), & y_{k B}=\max _{n}\left(y_{n}\right) \\ C\left(x_{k C}, y_{k C}\right), & x_{k C}=\min _{n}\left(x_{n}\right) \\ D\left(x_{k D}, y_{k D}\right), & y_{k D}=\min _{n}\left(y_{n}\right)\end{cases}
$$

For two trajectories corresponding to two signals, if the extreme points overlap (no matter the lag used), it is highly possible that their trajectories are similar (and so their corresponding signals), otherwise the signals are different. This parameter is very useful when it comes to study two dilated signals with the same amplitude.

In order to highlight the advantages brought by the points defined by (7), we determine their coordinates for lag $\tau=$ $1, \ldots, 25$ (half a period of $s[n]$ ), then compute the differences

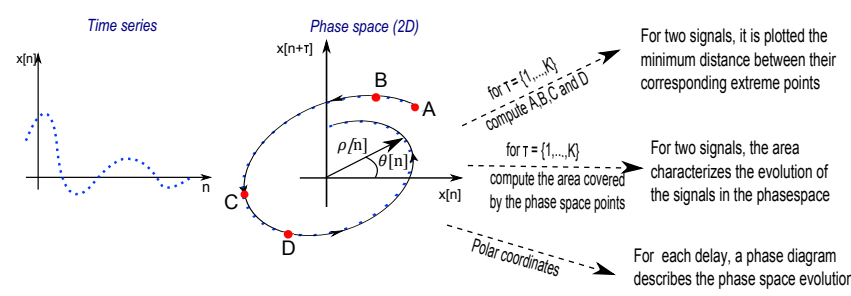

Figure 3: The schematic presentation of the multi-lag tools 


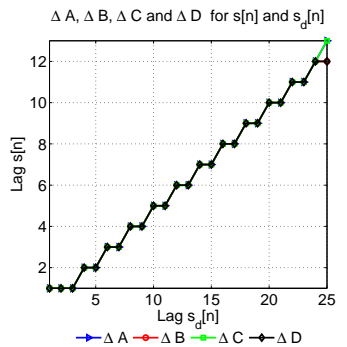

(a)

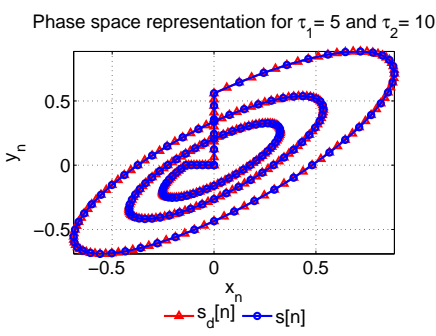

(b)

Figure 4: (a) The minimum difference between the extreme points on the phase space of $s[n]$ and $s_{d}$; (b) Phase space representation of $s[n]$ for lag $\tau_{1}=5$, respectively $s_{d}[n]$ for lag $\tau_{2}=10$.

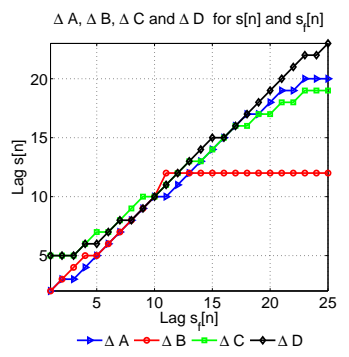

(a)

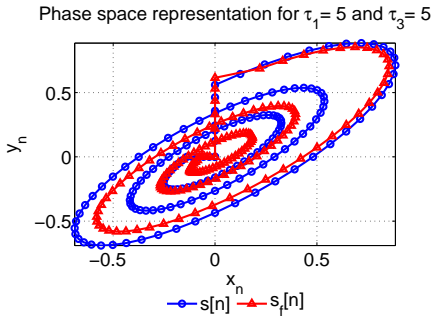

(b)

Figure 5: (a) The minimum difference between the extreme points on the phase space of $s[n]$ and $s_{f}$; (b) Phase space representation of $s[n]$ for lag $\tau_{1}=5$, respectively $s_{f}[n]$ for lag $\tau_{3}=5$

between them and, finally, we keep only the minimum values for each lag according to eq. (8).

$$
\Delta X=\min \left(X_{s_{1}}-X_{s_{2}}\right)
$$

where $X_{s_{i}}$ represents one of the extreme points of the trajectory from eq. (7) corresponding to the trajectory of signal $s_{i}, i=\{1,2\}$. The results are presented in fig. (4) and (5).

From fig. 4, it can be observed that the evolution of the trajectory of $s_{d}[n]$ is the same as the one of $s[n]$ when $\tau_{2} / \tau_{1}=$ 2 , emphasizing the hypothesis that $\alpha=2$. For this reason, the positions of these points overlap.

Concerning the evolution of $s_{f}[n]$, fig. 5(a) shows that the extreme points do not overlap perfectly. In the representation of the phase space for these signals, we choose $\tau_{1}=\tau_{3}=5$, due to the fact that there the positions of the extreme points start to partially overlap. It can be seen that the trajectories of the signals are different ( fig. 5(b)). It can be noticed that the amplitude difference and frequency shift has a major impact upon the evolution of the trajectory.

This observation leads to defining two more approaches: the elliptic modeling and the polar coordinates representation, respectively.

The use of the elliptic modeling is derived from [3]. The idea is based on the fact that the phase space evolution of a sine wave is an ellipse. We model the ellipse of the trajectory considering the solution that minimizes the system:

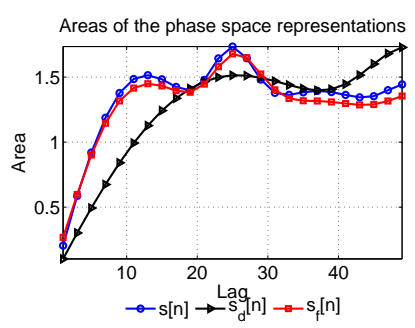

(a)

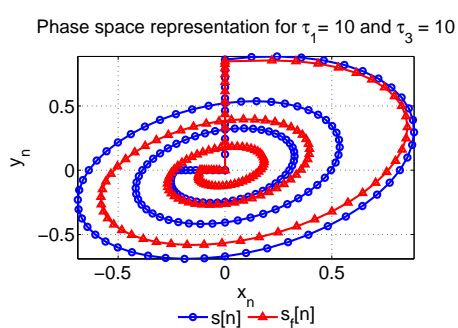

(b)
Figure 6: (a) The evolution of the area according to the lag; (b) the phase space representation for approximately the same value of the area $(A=1.35): \tau_{1}=10$ and $\tau=10$

$$
S=\sum_{i=1}^{M}\left[F\left(x_{i}, y_{i}\right)\right]^{2}
$$

where $F(x, y)=\Gamma x^{2}+\Lambda y^{2}+1$. A least square estimation is performed on the sum from eq. (9) resulting the pair $(\Gamma, \Lambda)$. It goes that the semi-major axis $a=1 / \sqrt{\Gamma}$ and the semi-minor axis $b=1 / \sqrt{\Lambda}$.

According to [5], [8], a lag that determines an average area describes a clear trajectory and excludes the cases of irrelevance or redundancy. Hence, after the elliptic modeling, we estimate the area of the circumscribed ellipse that includes the points on the trajectory.

Figure 6 highlights the evolution of the area in multi-lag representation. Obviously, that for the same value of the area for $s_{d}[n]$ and $s[n]$, the ratio between their lags is $\alpha=2$. Instead, it can be noticed that, although the lag-area evolutions have the same trend for $s_{f}[n]$ and $s[n]$, at higher lags, the values from $s_{f}[n]$ are slightly smaller than those of $s[n]$. In terms of evolution into the phase space, we have chosen a lag where the value of the area is different (fig. 6(b)). The phase space representation for the same value of the area (for $\tau_{1}=$ $\tau_{3}=5$ ) is given in fig. 5(b). The phase space representations are different regardless of the used lag. The shape of the ellipse is directly related to the frequency and amplitude content of the signal [5], [8].

Using the lags from the elliptic modeling, we represent the phase space trajectory in polar coordinates (eq. (10)).

$$
\begin{aligned}
& \varphi[n]=\sqrt{x_{n}^{2}+y_{n}^{2}} \\
& \theta[n]=\arctan \frac{y_{n}}{x_{n}}
\end{aligned}
$$

This representation is very useful for the study of signals having a certain amplitude variation. Figure 7 presents the evolution of $s_{d}[n]$ which is exactly the same as the one of $s[n]$. However, although $s_{f}[n]$ exibits the same number of oscillations as $s[n]$ (given by the number of curves on the phase diagram), the length of the vectors for negative angles is smaller than the corresponding vectors of $s[n]$. The lengths of the vectors for positive angles are also smaller than those of $s[n]$, except for the curve placed on top of the phase diagram. 


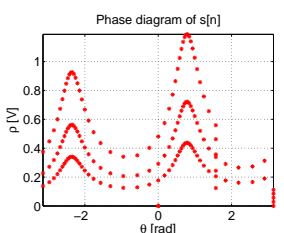

(a)

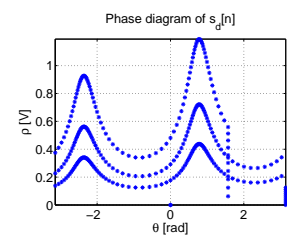

(b)

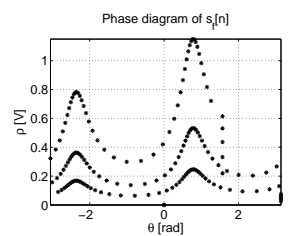

(c)

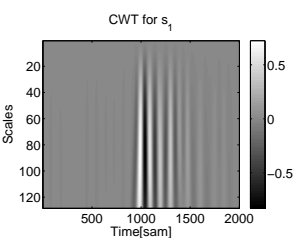

(a)

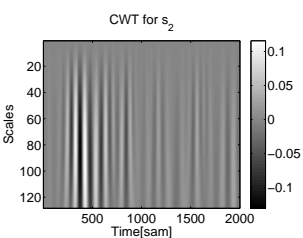

(b)

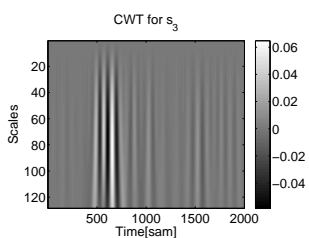

(c)
Figure 7: Polar coordinates representation: (a) $s[n]\left(\tau_{1}=5\right)$, (b) $s_{d}[n]\left(\tau_{2}=10\right),\left(\right.$ c) $s_{f}[n]\left(\tau_{3}=5\right)$

Figure 9: Percentage of energy for each wavelet coefficient: (a) $s_{1}$; (b) $s_{2}$; (c) $s_{3}$

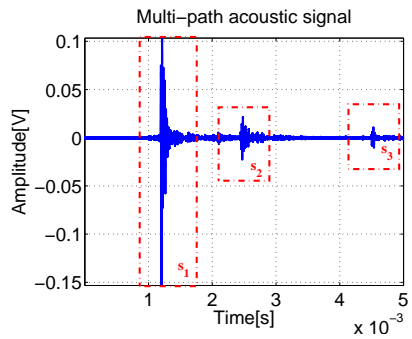

Figure 8: Multi-path acoustic signal recorded: $s_{1}$ - direct path; $s_{2}, s_{3}$ - other paths

This is directly related with the different amplitude modulation of the signals.

Moreover, the number of points between the negative angle peaks and the positive angle peaks are directly related to the fundamental frequency of the signal, which is, in fact, a semiperiod of the signal. Let $n_{0}$ be the number of points. It goes that:

$$
T_{0}=\frac{2 \cdot n_{0}}{f_{s}}
$$

where $T_{0}$ is the fundamental frequency of the signal and $f_{s}$ its sampling frequency.

Using eq. (11), we obtain $f_{s} / f_{s_{f}}=1.024$, pointing out when two signals are the same (using the extreme points), as well as to discriminating them (using the area and phase diagram).

\section{MULTI-PATH ACOUSTIC SIGNALS}

Considering the new information highlighted by these new tools of representation, we use them in the characterization of a multi-path acoustic signal, fig. 8 .

The signal was acquired with an acoustic microphone. It can be observed that $s_{1}$ is the signal arrived at the sensor on the direct path and $s_{2}, s_{3}$ are the reflexions of the acoustic signal arrived on other paths.

In fig. 9, we present the results obtained using the wavelet transform. Apart the signal amplitudes, there is no other discrimination between the multi-paths.

However, considering the tools presented in section 3, we used the elliptic modeling and determined the area of the circumscribed ellipse of the trajectory in the phase space. The signals are normalized to eliminate the area differences caused by the attenuation of $s_{2}$ and $s_{3}$. The results are presented in fig. 10 .

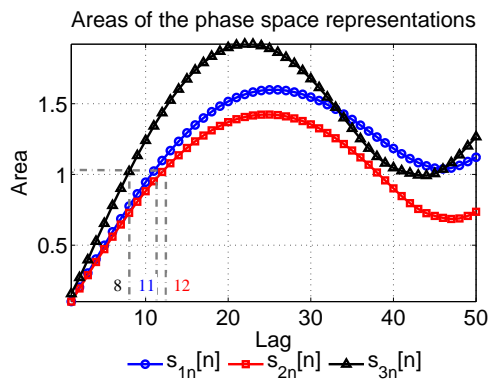

Figure 10: The multi-lag phase space representation of the area of the points from the trajectory

It can be noticed that the areas have different progress, although the signals are normalized. Still, their evolution seems to have the same trend. The choice of the lag is made so that the area of trajectories on the phase space maintains approximately the same average value $(A=1.02)$ : $\tau_{1}=11, \tau_{2}=12, \tau_{3}=8$. Figure 11 illustrates the phase diagram representation of the signals using the lags found in the previous paragraph.

It can be seen that the reflected signals are attenuated, because the norms of the reflexions $s_{2}$ and $s_{3}$ are significantly smaller that those of $s_{1}$. Moreover, it appears that the reflexions have fewer curves with peaks than the direct path signal, meaning that the reflexions bear fewer oscillations, which can be assigned to dispersion. The oscillations with an energetic level comparable to the noise are plotted on the thick area, therefore a discrimination between the relevant signal and the noise is hard to be obtained.

In addition, using the eq. (11), we obtain $f_{s_{1}} / f_{s_{2}}=0.94$ and $f_{s_{1}} / f_{s_{3}}=0.92$. Therefore, the reflected signals suffer a frequency shift. This is because of their propagation path where multiple phenomena take place: reflexion, diffusion,

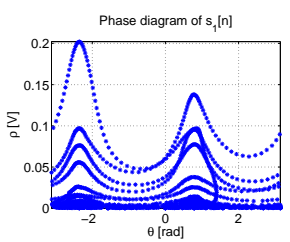

(a)

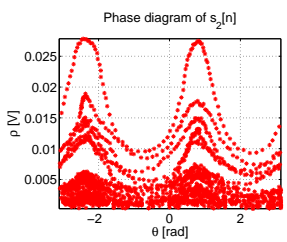

(b)

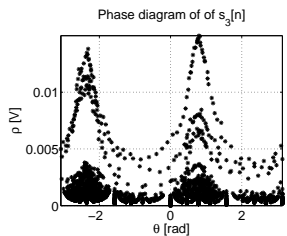

(c)
Figure 11: Polar coordinates representation for : (a) $s_{1}: \tau_{1}=$ 11 , (b) $s_{2}: \tau_{2}=12$, (c) $s_{3}: \tau_{3}=8$ 
dispersion, etc.

\section{CONCLUSION}

In the last years, transient signals have been the subject of major investigation and discussion in the signal processing community. While the transient signal detection is possible, their characterization/classification concerns a lot of application context, because of their effects that they can bring to the system according to their amplitude and/or frequency content.

As a follow-up of [3], the paper presents new methods of characterization and discrimination of transient signals. The methods are based on the concept of phase space representation which has its roots in the dynamical system theory. Firstly, the tools developed for the characterization are emphasized and their use is exemplified on simulated transitory signals. In the second part, these new concepts are applied on a multi-path acoustic signal. The extreme points method is able to highlight when two signals are alike. The elliptic modeling and the area provide important information for the discrimination and characterization of two similar signals. For the real signals, the methods were able to illustrate the change caused by different propagation paths.

Future work foresees the extension of the method for an $m$-dimensional phase space, working on several real transient signals and the exclusion of the noise drawbacks.

\section{ACKNOWLEDGEMENT}

This work has been supported by the Dema'Loc project funded by Institut Carnot "Energies du futur".

\section{REFERENCES}

[1] S. Mallat, S. Zhong, "Characterization o5f signals from multiscale edges", IEEE Transaction on Pattern Analysis and Machine Intelligence, vol. 14, No. 7, p. 710-732, July 1992.

[2] C. Strobl, P. Meckler, "Arc Faults in Photovoltaic Systems", Proc. 25th ICEC \& and 56th IEEE Holm Conference on Electrical Contacts, Charleston, 2010, pp. 216-222.

[3] C. Bernard, T. Petrut, G. Vasile, C. Ioana, "Multi-Lag Phase Space Representations for Transient Signal Characterization ", Europeean Signal Processing Conference, 2014, Portugal, pp. 2115 - 2119.

[4] J.-P Eckmann, S. Oliffson Kamphorst, and D Ruelle, "Recurrence Plots of Dynamical Systems," Europhysics Letters (EPL), vol. 4, no. 9, pp. 973-977, Nov. 1987.

[5] N. Marwan, S. Schinkel, J. Kurts, "Recurrence plots 25 years later Gaining confidence in dynamic transitions", Europhysics Letters, 101, 20007, 2013.

[6] Florin-Marian Birleanu, Cornel Ioana, Cedric Gervaise, Jocelyn Chanussot, Alexandru Serbanescu, and Gheorghe Serban, "On the recurrence plot analysis method behaviour under scaling transform," in 2011 IEEE Workshop on Statistical Signal Processing (SSP2011), June 2011, pp. 789-792.

[7] Florin-Marian Birleanu, Ion Candel, Cornel Ioana, Cedric Gervaise, Alexandru Serbanescu, and Gheorghe Serban, "A Vector Approach to Transient Signal Processing", in 11th International Conference on Information Science, Signal Processing and their Applications (ISSPA 2012), July 2012, pp. 1174-1179.

[8] C. Ioana, A. Digulescu, A. Serbanescu, I. Candel, F. M. Birleanu, "Recent advances in non-stationary signal processing based on the concept of recurrence plot analysis", Springer Proceedings in Mathematics \& Statistics: Translational Recurrences - From Mathematical Theory to RealWorld Applications, ISBN 978-3-319-09530-1, Springer International Publishing, Cham, 2014. 